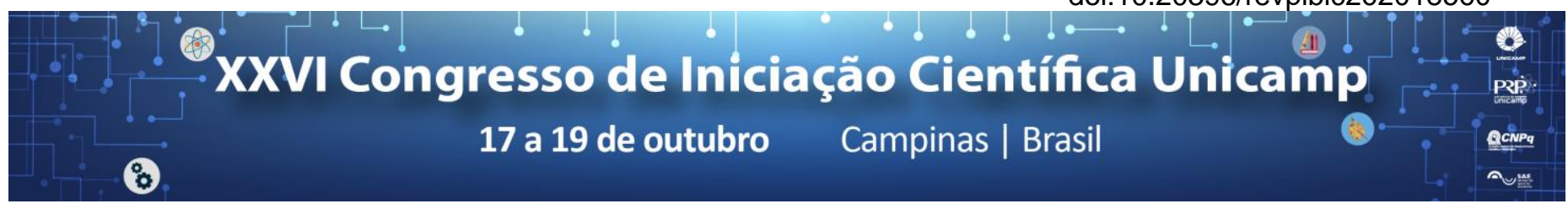

\title{
Study of Volt/VAr Control applied to Future Electrical Energy Distribution Systems
}

\author{
Guilherme O. Custódio*, Fernanda C. T. Arioli.
}

\begin{abstract}
Recently, several approaches have been proposed to mitigate the technical impacts caused by distributed generators in electrical energy distribution systems. Among these approaches one can detach the control scheme known as Volt/VAr, a centralized scheme that integrates voltage and reactive power control functions. In this context, this work aims to study the Volt/VAr control solved by an optimization tool, and focused on distribution systems with a high monitoring level and a massive integration of photovoltaic systems.
\end{abstract}

\section{Key words:}

Electrical Energy Distribution Systems, Optimal Power Flow, Voltage/VAr Optimization.

\section{Introduction}

The structure of Electrical Energy Distribution Systems (EEDS) is undergoing profound changes, becoming more monitored and controllable. The connection of distributed generators, for instance, photovoltaic (PV) systems, is one of these changes. With the increase in their number, it is possible that the voltages observed in the system surpass the statutory limits established by the Brazilian Electricity Regulatory Agency (ANEEL) and the power losses increase. This voltage violation, when attested, results in penalties to the utilities and losses to the customers that may have their devices damaged. Despite the negative technical impacts, the inverters of PV systems can be used to aid voltage and reactive power regulation. In this context, this work aims to study the Volt/VAr control, a centralized scheme that integrates voltage and reactive power control functions, solved by an optimization tool, AIMMS1.

\section{Results and Discussion}

The EEDS used in the tests presented in this work is IEEE 13 Node Test Feeder ${ }^{2}$, which has 15 customers, 2 transformers - one is an on load tap changer (OLTC) transformer with 32 taps - and a three-phase capacitors bank with 6 taps. In addition, PV systems are installed to the EEDS according to a given penetration level, defined as a percentage of total customers. In this work, the optimal power flow (OPF) is the basis of the centralized control scheme. Hence, a three-phase OPF model was developed in AIMMS in order to analyze unbalanced EEDS. In this model, the loads are represented by their active and reactive power, the three-phase capacitors bank as a reactive power injection, which depends on the state defined by its tap, and PV systems are modeled based on active power and their power factor (PF), which changes according to their injection/absorption of reactive power. The objective function is to minimize losses by active power in lines, being the controllable variables the taps of OLTC transformer and three-phase capacitors bank as well as the points that define the Volt-VAr curve of each PV system. When active, the centralized control scheme checks every 15 minutes if there is any voltage violation, i.e., if the customer voltages are not within $0.920 \mathrm{pu}$ and $1.050 \mathrm{pu}$, thresholds determined by $\mathrm{ANEEL}^{3}$. When any violation is found, the centralized control scheme determines the optimal state for the network at that moment in order to restrict the voltages to these limits. After that, the controllable variables are updated in the real EEDS according to the optimal solution obtained. Image
1 (a) shows the highest voltages of all customers observed during the one-day period with centralized control inactive and Image 1(b) the highest voltages of all customers observed during the same period with centralized control active. Both scenarios are simulated with $50 \%$ PV systems penetration level. In the former case, customer voltages reach $1.098 \mathrm{pu}$, surpassing the upper threshold, $1.050 \mathrm{pu}$, during $40 \%$ of the day. In the latter, the voltages remained within the established thresholds during $99 \%$ of the day and the highest customer voltage observed is $1.051 \mathrm{pu}$.

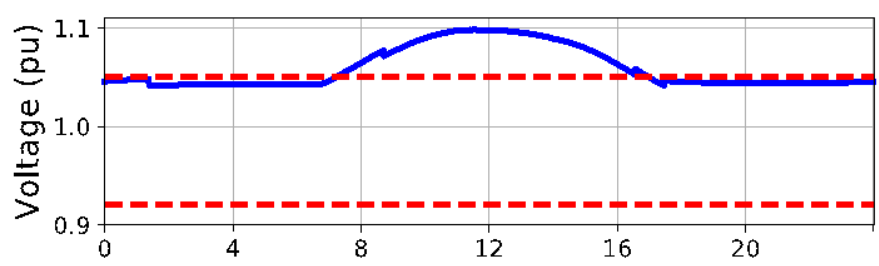

(a)

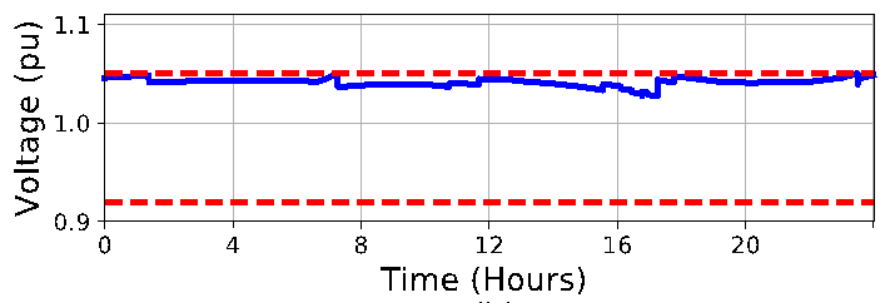

(b)

Image 1. Highest customer voltages along the day with centralized control inactive in (a) and active in (b).

\section{Conclusions}

The optimization model assumes the role of the centralized control and minimizes active power losses considering the presence of PV systems, taking into account voltage thresholds determined by ANEEL. The results show the potential of that concept, especially when applied to future electrical energy distribution systems.

\section{Acknowledgement}

This work was supported by São Paulo Research Foundation (FAPES) grant \#2017/21058-8.

\footnotetext{
${ }^{1}$ AIMMS - Advanced Interactive Multidimensional Modeling System. [Online]. Available in: <https://aimms.com>. Accessed: July, 2018.

${ }^{2}$ IEEE, "13-bus Feeder". [Online]. Available in: < http://sites.ieee.org/pestestfeeders/resources/>. Accessed: July, 2018.

${ }^{3}$ ANEEL - Agência Nacional de Energia Elétrica. PRODIST: Módulo 8. [Online]. Available in: 〈http://www.aneel.gov.br/modulo-8>. Accessed: July, 2018.
} 